\title{
Factores que influyen en la calidad y cantidad de Responsabilidad Social en las empresas españolas. Estudio de caso de las empresas del Ibex 35
}

\author{
Orencio Vázquez Oteo
}

\section{RESUMEN}

Las empresas han pasado de ser meros actores económicos a ser determinantes en el marco de las relaciones políticas y sociales, nacionales e internacionales. Su mayor tamaño, unido a la deslocalización de los centros de producción, dificulta el control por parte de los países que anteriormente disponían de mayor facilidad para fiscalizar el comportamiento de las empresas. La responsabilidad social trata de dar respuesta a estos desafíos y generar un marco de normas y principios por los que debe regirse el comportamiento de las empresas en su búsqueda del beneficio.

Las empresas responden ante este nuevo escenario adoptando compromisos y políticas en el ámbito social, económico, medioambiental y de buen gobierno. La mayor parte de las empresas cotizadas presentan informes de sostenibilidad. El presente artículo expone los resultados de una investigación que trata de extraer conclusiones relevantes acerca de qué factores influyen en la presentación de la información no financiera por parte de las grandes empresas españolas cotizadas, más concretamente de las empresas del lbex 35 durante el periodo de 2005 al 2011.

PALABRAS CLAVE: Responsabilidad social corporativa, rendición de cuentas, calidad de la información, motivaciones para información no financiera.

CLAVES ECONLIT: M140, M100, G320.

Cómo citar este artículo: VÁZQUEZ, O. (2015): "Factores que influyen en la calidad y cantidad de Responsabilidad Social en las empresas españolas. Estudio de caso de las empresas del lbex 35", CIRIEC-España, Revista de Economía Pública, Social y Cooperativa, 85, 181-215.

Correspondencia: Orencio Vázquez Oteo, profesor asociado de la UNED.

E-mail: ovazquez@observatoriorsc.org. 


\section{Les facteurs qui influencent la qualité et la quantité de responsabilité sociale dans les entreprises espagnoles. Étude de cas concernant les entreprises cotées à l'lbex 35}

RÉSUMÉ : Les entreprises sont passées de simples acteurs économiques à être déterminantes dans le cadre des relations politiques et sociales, nationales et internationales. Le volume plus important des entreprises, ainsi que la délocalisation des centres de production rendent difficile le contrôle de la part des gouvernements nationaux qui, auparavant, disposaient d'une plus grande facilité pour soumettre le comportement des entreprises à un contrôle fiscal. La responsabilité sociale essaie de répondre à ces défis en générant des normes et des principes qui devraient régir le comportement des entreprises dans leur recherche de profit.

Les entreprises répondent à ce nouveau scénario en adoptant des engagements et des politiques dans le domaine social, économique, environnemental et de bonnes pratiques de gestion. La plupart des entreprises cotées présentent des rapports de développement durable. Cet article expose les résultats d'une recherche visant à tirer des conclusions pertinentes concernant les facteurs qui influencent la présentation des informations non financières provenant des grandes entreprises espagnoles cotées, en particulier les entreprises de l'lbex 35 , au cours de la période 2005-2011.

MOTS CLÉ : Responsabilité sociale des entreprises, reddition de comptes, qualité de l'information. Motivations concernant les informations non financières.

\section{Factors that influence the quality and quantity of social responsibility in Spanish companies. A case study of IBEX 35 companies}

ABSTRACT: Companies have ceased to be mere economic agents and have become determining factors in the context of national and international political and social relations. Their larger size, along with the relocation of production centres, makes them harder to control for countries that previously found it easier to oversee their behaviour. Social responsibility tries to provide an answer to these challenges and to generate a framework of rules and principles that should govern the behaviour of companies in their pursuit of profit.

Companies respond to this new scenario by adopting policies and giving undertakings in the social, economic, environmental and good governance spheres. Most listed companies publish sustainability reports. This article focuses on the results of an investigation that aimed to extract relevant conclusions about the factors that influenced the publication of non-financial information by large listed Spanish companies, specifically lbex 35 companies, during the 2005 to 2011 period.

KEY WORDS: Corporate social responsibility, accountability, quality of information, motives for providing non-financial information. 


\section{1.- Introducción}

La responsabilidad social corporativa (RSC) pivota sobre tres principios básicos, que son la inclusividad (basado en la visión multistakeholder, orientación a las partes interesadas), el de estar fundamentada sobre los impactos económicos, sociales y ambientales en las partes interesadas (derivados de acciones u omisiones decididas), y el de la rendición de cuentas de las actuaciones, resultados e impactos (a los stakeholders o partes interesadas y a la sociedad en general). Una comunicación efectiva con los grupos de interés es un instrumento importante para generar confianza y lealtad (Wheeler y Sillanpaa, 1998).

De la evolución en el número de informes de responsabilidad social o sostenibilidad durante la última década y de la cobertura de los temas relacionados con la transparencia y rendición de cuentas de la información no financiera en los debates institucionales y medios de comunicación, se desprende el interés suscitado tanto por parte de la empresa como por parte de los diversos grupos de interés, en la materia. Durante este periodo también se han multiplicado las investigaciones académicas que, por un lado, tratan de evaluar los factores que son determinantes en la cantidad y calidad de la información emitida por las empresas y, por otro, ponen en evidencia que la información todavía dista de responder a un proceso de rendición de cuentas.

Los estudios e investigaciones en este campo se apoyan en diferentes perspectivas teóricas para justificar o fundamentar la presentación de información social, medioambiental y de buen gobierno por parte de la empresa. Estas teorías van desde la de la legitimidad, la teoría de los stakeholders, la de la agencia y de contabilidad positiva, entre otras. Sin embargo y pese a la cantidad de estudios existentes, no hay un acuerdo unánime sobre el factor o factores que influyen de manera determinante en que la empresa presente información social, medioambiental y de buen gobierno. Por un lado, se considera fundamental el marco institucional en donde la empresa desarrolla su actividad y, por otro, las características propias de la empresa como capitalización, beneficios, industria a la que pertenecen, país origen de la propiedad o cualidades de los directivos.

En España, existen diversas investigaciones que tratan de explicar los factores determinantes en que la empresa facilite información no financiera, no obstante la mayor parte de las mismas se centran en evaluar la información parcial, normalmente de carácter medioambiental.

En el presente trabajo se expone la evolución de la información social, medioambiental y se realiza una revisión de la literatura existente en relación a los factores que determinan que las empresas provean a los mercados y a los diferentes grupos de interés información social y medioambiental. 
En una segunda parte del artículo se exponen los principales resultados de una investigación que evalúa los factores que son determinantes en la presentación de información extra financiera por parte de las empresas que componen el selectivo español lbex 35 tomando como base la teoría de la legitimidad.

\section{2.- La transparencia y comunicación en RSC}

\subsection{Transparencia como elemento clave de la RSC y generador de confianza}

De acuerdo con Hackston y Milne (1996: 77) reducir las presiones por parte de diversos ámbitos ha influido en la asunción por parte del sector privado de una responsabilidad de los impactos que causan en la sociedad producto de sus actividades. Por ello se insta a las empresas a ser responsables y rendir cuentas a un público más amplio que a accionistas y acreedores.

La transparencia se ha convertido, de acuerdo con Global Reporting Iniciative (GRI) en un elemento crítico para construir confianza, mantener o mejorar la reputación y la gestión de riesgos (GRI, 2015: 4). La mejor estrategia para generar confianza debe estar sustentada en los pilares de la transparencia, la participación y la rendición de cuentas. A través de la transparencia, la empresa puede transmitir la idea de que forma parte de las sociedades de manera responsable y que, por tanto, está comprometida a afrontar sus retos y alcanzar sus metas conjuntamente con el resto de actores que las integran.

El estudio "State of Sustainable (2014)" realizado por la empresa The Business for a Better World basado en una encuesta con una muestra de más de 700 personas mayoritariamente directivos de empresas (92\%) de todo el mundo, la mayoría de los encuestados (61\%) menciona la transparencia como mejor herramienta para recuperar la confianza.

Desde una visión de gestión de la relación con las partes interesadas, informar sobre las medidas adoptadas por la empresa para minimizar las externalidades negativas sobre los grupos de interés ayudará a ambas partes, empresas y grupos afectados, a incrementar su grado de conocimiento. Como afirma Patten (1992) el reconocimiento de unos participes con intereses en la empresa, diferentes a accionistas y acreedores, exige no solamente comportamientos capaces de satisfacer a los diversos agentes o stakeholders, sino también una política informativa que permita visualizar la asunción de esos compromisos. Los compromisos, acciones y políticas en el ámbito de la responsabilidad social corporativa requieren comunicar por parte de la empresa para obtener un reconocimiento del mercado que al mismo tiempo se traduzca en una ventaja competitiva. MacWilliams y Siegel (2001) plantean 
que para que la RSC sea un elemento diferenciador los clientes deben ser conscientes de las acciones emprendidas por la empresa en este ámbito.

La transparencia es un elemento clave de la RSC y en la construcción de la reputación de la empresa. Son varios los estudios que lo ratifican: El estudio "Value of sustainability reporting In the 2013" realizado por el Boston College Center for Corporate Citizenship y la empresa Building a Better World (2014) en base a una encuesta a 579 empresas señala la mejora de reputación como el factor que más valor genera el informe de sostenibilidad". El estudio "Report on the Scope and Quality of CSR Reports from the World's Largest Companies" elaborado por investigadores del Weissman Center for International Business at Baruch College (2014) señala que una alta calidad en el informe de sostenibilidad puede tener un papel importante en la construcción de la reputación de la empresa. El estudio "State of Sustainable (2011)"2 realizado por la empresa The Business for a Better World y GlobeScan en base a una encuesta a 498 profesionales en su mayoría directivos de empresas, afirma que la transparencia es la mejor herramienta para mejorar la confianza en la empresa. Los encuestados son pesimistas acerca del nivel de confianza en la empresa privada, la mayor parte de las respuestas señalan que la sociedad tiene muy baja confianza en los negocios.

\subsection{Triple cuenta de resultados. El informe de sostenibilidad}

La comunicación de la RSC por parte de la empresa es utilizada a través de diversos canales que van desde blog, redes sociales, web corporativas, webs creadas expresamente y campañas de publicidad, entre otros. Pese a la multiplicidad de herramientas de comunicación promovida por el desarrollo de las nuevas tecnologías, es el informe de sostenibilidad la herramienta que las organizaciones más utilizan para comunicar e informar sobre su comportamiento y el resultado de la gestión de impactos sociales y medioambientales.

Dentro de los diversos canales de comunicación, el informe de sostenibilidad es el medio utilizado por la empresa que más se acerca a un proceso sistemático de rendición de cuentas. En la actualidad, el informe de sostenibilidad o RSC está asociado al término acuñado por Elkington (1997): "triple cuenta de resultados (TCR)" que consiste en la elaboración de un informe que atienda a las demandas de sus grupos de interés a través integrar de manera equitativa y equilibrada los resultados económicos, sociales y medioambientales. Para el autor, la TCR es clave en la sostenibilidad y ha supuesto un cambio en la mentalidad de los empresarios pasando de una situación de pánico hacía el ecologismo a una asunción del concepto de ecoeficiencia. Para Elkington y los promotores de la TCR la novedad consiste en contemplar tres tipos de capital: no solamente el capital financiero, sino también el natural y el humano (Rambaud y Richard, 2015: 4). Pese al apoyo mayoritario al planteamiento

1.- $h$ ttp://www.ey.com/Publication/vwLUAssets/EY_-_Value_of_sustainability_reporting/\$FILE/EY-Value-of-Sustainability-Reporting.pdf 2.- $h$ ttp://www.bsr.org/reports/BSR_GlobeScan_Survey_2014.pdf

http://www.bsr.org/reports/BSR_Globescan_State_of_Sustainable_Business_Poll_2011_Report_Final.pdf 
de Elkington, hay críticas a la TCR basadas entre otros aspectos en vaguedad del concepto y su definición, que se puedan ocultar resultados financieros detrás de datos 0 cifras sociales o medioambientales y la falta de efectividad en relación a cambiar realmente la visión y el comportamiento dentro de la empresa (Norman y MacDonald, 2004; Rambaud y Richard, 2015; Milne y Gray, 2013).

Gray et al. (1987: 9) define la contabilidad social y medioambiental como "El proceso de comunicar los efectos sociales y ambientales de las acciones económicas de las organizaciones a determinados grupos de interés dentro de la sociedad, y para la sociedad en general". Tagesson et al. (2009: 352) define la información social como "el informe que considera aspectos medioambientales, éticos y sociales".

De acuerdo con GRI (2011: 3), el informe de sostenibilidad es "la práctica de la medición, la divulgación, y de rendir cuentas a los grupos de interés internos y externos sobre el desempeño organizacional hacia el objetivo de un desarrollo sostenible. El informe de sostenibilidad es un término amplio sinónimo de otros utilizados para describir la presentación de informes sobre los impactos económicos, ambientales y sociales (por ejemplo, triple cuenta de resultado, informes de responsabilidad corporativa, etc.). Un informe de sostenibilidad debe proporcionar una representación equilibrada y razonable del desempeño de la sostenibilidad de una organización, incluyendo tanto las contribuciones positivas como negativas".

\subsection{Evolución de los informes de sostenibilidad}

Las empresas comienzan a publicar informes sociales y medioambientales mucho tiempo atrás, por ejemplo Hogner (1982) nos muestra cómo los primeros informes financieros de las empresas del acero estadounidenses incluían aspectos como desarrollo comunitario, beneficios de los trabajadores o seguridad en el lugar de trabajo. Pero es a principios de los años 70 cuando de alguna manera se empieza a popularizar la elaboración de informes sociales a partir de las preocupaciones acerca de los derechos civiles, como los derechos de la mujer, los problemas raciales y el anti belicismo. En los años 70 y 80 del S.XX la mayor parte de los estudios se refieren a informes sociales que habitualmente se presentan dentro de los informes financieros. En los años 90 el informe medioambiental es el que centra mayor atención. Fruto de los desastres medioambientales provocados por empresas como el caso de Bhopal en la India que causó 20.000 muertes y al menos 600.000 afectados por una fuga química de una planta de la empresa Union Carbine (Observatorio de RSC, 2009), se producen presiones sociales y regulatorias para que las empresas informen sobre sus impactos medioambientales y la gestión de los mismos. Tal es el caso de US Emergency Planning and Community Right-To-Know Act en 1986, que requiere a las empresas que publiquen información sobre más de 320 sustancias químicas, en Alemania con la aprobación en 1991 de la Ordinance on the Avoidance of Packaging Waste bajo la German Waste Act o la exigencia por parte del Gobierno Danés que desde 1999 requiere a ciertas empresas que informen sobre las consecuencias medioambientales de su actividad (Soderstrom, 2013: 32). Después del cambio de milenio la terminología predominante cambio a 
informe de responsabilidad social corporativa o informe de sostenibilidad (Fifka, 2012; Soderstrom, 2013). En los últimos años (2008-2014) se ha impuesto la denominación de informe de sostenibilidad sobre la nomenclatura informe de responsabilidad social corporativa. De acuerdo con el estudio "The KPMG Survey of Corporate Responsibility Reporting 2013" (KPMG, 2014) realizado sobre una muestra de 4.100 empresas en todo el mundo, el $43 \%$ de las empresas lo denominan informe de sostenibilidad mientras que sólo un $25 \%$ lo denomina informe de responsabilidad social corporativa.

Desde los años 70 del siglo pasado a los momentos actuales (2015) el número de informes de sostenibilidad publicados por empresas ha crecido exponencialmente como respuesta a la demanda de información ambiental, social y de buen gobierno (ASG) planteada desde reguladores, agencias de rating, bolsas de valores, consumidores, inversores y organizaciones de la sociedad civil. En el año 1996 de acuerdo con estimaciones de Sustainability y Unep (1997) el número de informes medioambientales o de sostenibilidad ascendían aproximadamente a 350. KPMG realizó en 1993, 1996 y 1999 ediciones sucesivas del estudio "KPMG international survey of enviariomental reporting". De acuerdo con KPMG el número de empresas sobre una muestra de 1.193 empresas que tenían informe medioambiental o de sostenibilidad, ha pasado del 13\% en 1993, el 17\% en 1996 y el 24\% en 1999. Posteriormente KPMG realiza el estudio "KPMG, international survey of Corporate Social Responsibility" en ediciones de 2002, 2005, 2008, 2011 y 2013. De acuerdo con KPMG de las 100 mayores empresas en una muestra amplia de países (en la edición de 2013 incluye a 4.100 empresas de 41 países) el porcentaje de empresas que presentan informes de sostenibilidad ha pasado del $24 \%$ en 2002 al $71 \%$ en 2013. Para las 250 mayores empresas del mundo el porcentaje pasa del $45 \%$ en 2002 al $93 \%$ en 2013:

\section{Gráfico 1. Evolución porcentaje de empresas que presentan información ASG}

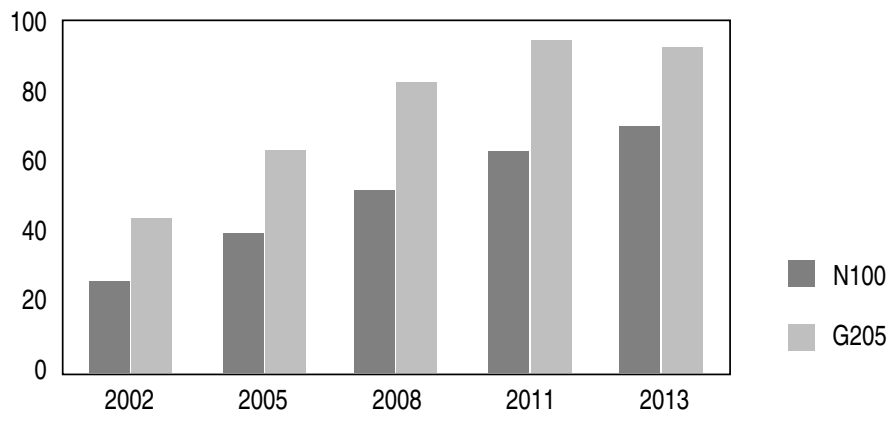

FUENTE: KPMG. 
De acuerdo con un análisis realizado por la empresa Governance \& Accountability Institute, INC (2014), el número de empresas del índice bursátil S\&P 500 que publican informes de sostenibilidad o RSC en 2014 asciende al 75\% del total de empresas incluidas en el S\&P 500. En 2011 esa cifra era de solamente el $20 \%$ de las empresas.

CorporateRegister.com, base de datos de informes de sostenibilidad on line, cifra en 7.920 las empresas que han publicado informe de sostenibilidad en 2014:

\section{Gráfico 2. Número de empresas que publican informes de sostenibilidad}

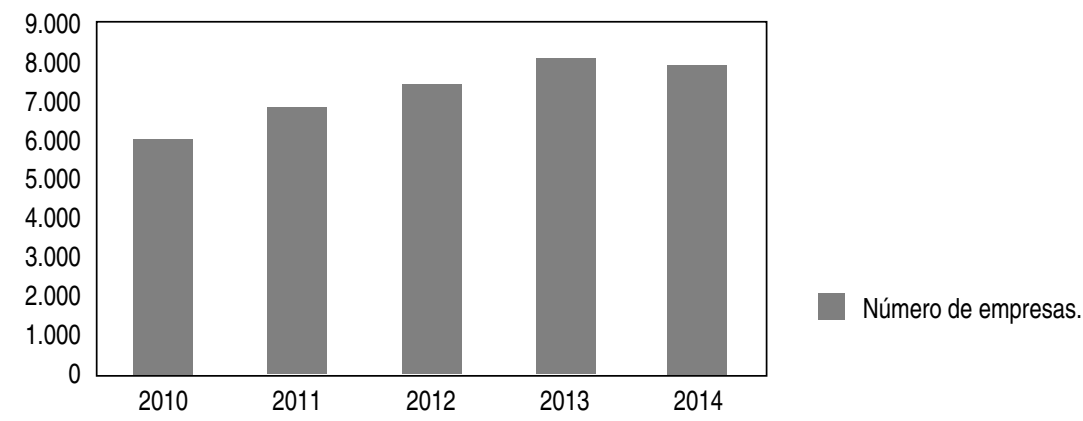

FUENTE: Corporateregister.com.

El estudio "State of Sustainable (2014)"3, realizado por la empresa The Business for a Better World (2014), basado en una encuesta con una muestra de más de 700 personas mayoritariamente directivos de empresas $(92 \%)$ de todo el mundo, señala a la transparencia como el tercer factor que más influencia tendrá en la empresa en los próximos 24 meses, solamente por detrás de los cambios tecnológicos y los cambios económicos.

En base a los datos facilitados por diversas fuentes acerca de la importancia de la transparencia y la evolución del número de empresas que presentan informes de sostenibilidad o RSC se puede afirmar que, si bien la presentación de tipo social y medioambiental, es algo reciente la tendencia apunta a que en pocos años este tipo de información será suministrada por la mayor parte de las empresas de cierto tamaño y/o que coticen en mercados organizados. 
En 2004 se introduce un nuevo concepto: el principio de materialidad que va a marcar el desarrollo futuro de estándares de información en RSC y los requerimientos exigidos por parte de algunos gobiernos. El concepto de materialidad es derivado del campo de la auditoría financiera que es definida como "la magnitud de una omisión o tergiversación de la información contable que, a la luz de las circunstancias, hace que sea probable que la decisión de una persona razonable habría cambiado o se habría visto influenciada por la omisión o declaración errónea" (Finantial Accounting Standards Board, 2008: 6).

GRI incorpora la materialidad como principio en su guía GRI 3, publicada en 2004. GRI describe la materialidad como "aquellos aspectos e indicadores importantes a la hora de reflejar los impactos sociales, ambientales y económicos de la organización, o influencien las decisiones de los grupos de interés... En la información financiera, la materialidad normalmente se considera un umbral a partir del cual debe ser tenido en cuenta en las decisiones económicas de los usuarios de los estados financieros de una organización (de los inversores, en especial). Este concepto de umbral también es importante en la elaboración de memorias de sostenibilidad, pero hace referencia a una gama mayor de impactos y de grupos de interés. La materialidad en el contexto de la sostenibilidad no se limita sólo a aquellos aspectos de la sostenibilidad que tienen un impacto financiero importante en la organización. Determinar la materialidad en una memoria de sostenibilidad también implica tener en cuenta los impactos sociales, ambientales y económicos que superan ese umbral, afectando a la capacidad de satisfacer las necesidades del presente sin comprometer las necesidades de las generaciones futuras" (GRI, 2004: 10)

Para Sustainability Accounting Standards Board (SASB) la materialidad implica aquella información que es importante para que el inversor tome decisiones de inversión. Para Lydenberg et al (2010) hay un reconocimiento de que los informes financieros no reflejan los intangibles responsables del $80 \%$ del valor de la empresa. Para SASB la información social y medioambiental es importante para el inversor porque, entre otras razones, los inversores institucionales tienen una responsabilidad fiduciaria que conlleva considerar como material información no financiera y los impactos sociales y medioambientales negativos provocados por una empresa tienen costes materiales para los inversores, las empresas y la sociedad en su conjunto que no se encuentran recogidos en los informes financieros. SASB ha elaborado un "mapa de materialidad" para siete sectores de actividad, que en un futuro alcanzaran a 88 industrias, sobre 30 temas divididos en cinco dimensiones: Medioambiente, capital social, capital humano, modelo de negocio e innovación, y liderazgo y buen gobierno.

En mayo de 2013, Global Reporting Iniciative publica su cuarta generación de guía para el informe de sostenibilidad (G4), en las que ocupa un lugar destacado la materialidad como núcleo del proceso de información. G4 requiere a las organizaciones una lista de sus temas materiales, el proceso llevado a cabo para determinar qué es material y las partes relevantes de la organización o de la cadena de valor donde los temas materiales son aplicables. Además, la empresa debe evaluar con regularidad los aspectos relacionados con la materialidad, tener en cuenta a las partes interesadas, tanto internas como externas, a la hora de evaluar la materialidad e informar de cómo gestiona los asuntos que se han determinado como materiales para mejorar su rendimiento en el tiempo. 
En conclusión, no existe una definición universalmente aceptada de materialidad lo que conlleva riesgos en cuanto a la calidad y comparabilidad de la información suministrada. Además, las diversas definiciones de materialidad están condicionadas por el destinatario objetivo de la información: Inversores, reguladores u otros grupos de interés. Puede haber coincidencias en cuanto a la información que esperan de la empresa entre los distintos grupos de interés pero también pueden generarse conflictos entre los mismos en base a sensibilidades y demandas diversas y no convergentes.

La última tendencia a partir de la creación en 2010 del International Integrated Reporting Committee (IIRC) en la provisión de información ASG, es integrar los aspectos relativos a la sostenibilidad con la información financiera, lo que se denomina informe integrado. Con carácter previo al IIRC, the King Code of Governance Principles and the King report on Governance (King III), exige a las empresas que cotizan en el mercado de Johannesburgo que presenten de manera integrada los aspectos relativos a buen gobierno, estrategia y sostenibilidad.

El informe integrado responde de alguna manera a la necesidad de los inversores y otros grupos de interés de que las empresas proporcionen resultados en todas las dimensiones de su actividad -social, ambiental, económica y de gobierno corporativo-, que esa información esté conectada y permita identificar los riesgos y oportunidades desde una perspectiva de sostenibilidad y del modelo de negocio desarrollado. El estudio realizado por $\operatorname{ACCA}^{4}$ y Eurosif 5 (2013) "What do investors expect from non-financial reporting? ?" basado en una encuesta realizada a 94 instituciones financieras en 18 países señala que el $92 \%$ de los inversores encuestados opinan que la información financiera y no financiera debería estar más integrada. The KPMG Survey of Corporate Responsibility Reporting 2013 (KPMG, 2014) encuesta realizada sobre una muestra de 4.100 empresas en todo el mundo, señala que pese a un número creciente de empresas comienzan a incluir información ASG, en su mayoría como un capítulo autónomo, en sus informes financieros, solamente un 3\% publica información financiera y no financiera de manera integrada.

El informe integrado es una práctica contable que persigue gestionar nuevos riesgos que afrontan las empresas (Miller et al. 2008). El informe integrado se basa en principios contables como evidencia sólida y fiable en cuanto a la recolección cuantitativa de datos, la materialidad, fiabilidad, comparabilidad y aseguramiento, para traducir el discurso de la sostenibilidad en un "lenguaje" comprensible para aquellos que toman decisiones sobre la organización (Thomson, 2015). Para Thomson (2015: 966) el potencial del IR es limitado debido a que desde su creación está instalado en la filosofía del "Business case" en lugar de una aproximación de "sustainability case" para las empresas.

4.- www.accaglobal.com

5.- www.eurosif.org

6. - http://www.accaglobal.com/content/dam/acca/global/PDF-technical/sustainability-reporting/tech-tp-wdir.pdf 


\subsection{Comunicación o rendición de cuentas}

La comunicación desde una perspectiva de la RSC, en línea con el punto de vista instrumental, se vincula a menudo con marketing, relaciones públicas o gestión de la reputación. De acuerdo con el punto de vista político-normativo la comunicación en RSC es entendida como un diálogo orientado al consenso que crea una comprensión compartida de la RSC (Schultz, F., et al 2013)

Desde una perspectiva de rendición de cuentas y aunque el término no está dotado de un significado preciso y depende de la disciplina o materia a la que haga referencia, en responsabilidad social corporativa se puede definir como "la responsabilidad de informar sobre aquellas acciones y compromisos de los que uno se hace responsable" (Gray, Owen, y Adams, 1996: 38). Los receptores de la información ASG o aquellos públicos objetivos a los que la empresa debe rendir cuentas serán: accionistas, consumidores, ONG, empleados, gobiernos locales y otras administraciones, proveedores, analistas, inversores y otros grupos que afecten o se vean afectados por la empresa. Gray et al (1997) se plantean quién decide la información adecuada y suficiente para los diversos grupos de interés y cómo sería el proceso de selección de ésta información. Para estos autores la teoría de los stakeholders no resuelve esta pregunta. Según esta teoría sería la empresa la que finalmente seleccionaría la información. Esta información normalmente no es relevante, salvo que sea asumida moralmente por la organización, cuestión que desechan ya que las "fuerzas del mercado" favorecen que se presente información solamente cuando ésta sea en interés o beneficie a la empresa. Desde una perspectiva de la teoría contable la información además de venir determinada por las partes interesadas, que puede verse sesgada por la preponderancia de uno de los grupos de interés o condicionada por los deseos de la empresa, debe establecerse en base a la demanda de la sociedad en su conjunto. Aquella información que sea de interés para la sociedad en su conjunto deberá proporcionarse por la empresa, de manera independiente a la demanda o negociación entre partes. Estas demandas o necesidad de información pueden abarcar o encuadrarse en marcos de tipo legal o normativo o de tipo ético o moral. Para los autores, las preocupaciones de tipo ético o moral deben ser recogidas por el derecho positivo. Finalmente y como tercera capa complementando la teoría de los stakeholders y la teoría contable, Gray et al (1997) nos hablan de la "polyvocal citizenship perspective" (PCP). Esta teoría está influenciada por los trabajos de NEF y Traidcraf, y enfatiza en dar prioridad a la voz de las partes interesadas a través del continuo diálogo con las mismas con reuniones periódicas. En cualquier caso, para que realmente haya un consenso y visión compartida de la RSC, la rendición de cuentas en RSC conlleva capacitar a los grupos de interés para que estos puedan exigir cuentas a la empresa y utilizar la información proporcionada a través del empoderamiento y la comunicación con ellos (Bailey et al., 2000; Cooper y Owen, 2007).

Un aumento en la cantidad y tamaño de los reportes no implica un aumento en su calidad y, pese a los avances realizados, cabe preguntarse en qué medida la información de aspectos sociales, ambientales y de gobierno corporativo responde a un verdadero compromiso de la empresa para rendir cuentas de sus actividades e impactos ante sus grupos de interés; 0 se trata simplemente de información utilizada para dar una buena imagen de la compañía, al centrarse en mostrar aquellos aspectos que 
pueden ayudar a la reputación de la empresa y no a los requisitos intrínsecos a la RSC de responder de una forma transparente y amplia sobre los aspectos realmente materiales para sus grupos de interés. Bailey et al (2000) analizan informes de sostenibilidad de varias empresas del Reino unido y concluyen que en prácticamente todas ellas hay un compromiso de rendición de cuentas con sus grupos de interés e incluso mencionan canales de comunicación con los mismos, pero sin embargo esta retórica no se traslada a evidencias de que lo anterior se traduzca en procesos de gestión dentro de la compañía y como resultado haya una verdadera rendición de cuentas. De acuerdo con el estudio Survey of Corporate Responsibility Reporting 2013 (KPMG, 2014) realizado sobre una muestra de 4.100 empresas en todo el mundo, solamente un $5 \%$ de las 250 empresas cotizadas de mayor tamaño informan sobre la manera de evaluar los asuntos materiales de manera continuada. El estudio realizado por ACCA7 y Eurosif (2013) "What do investors expect from non-financial reporting?" basado en una encuesta realizada a 94 instituciones financieras en 18 países señala que el $84 \%$ de los inversores están en desacuerdo o fuertemente en desacuerdo con que las empresas identifican de manera clara los aspectos materiales.

Según diversos autores e instituciones los informes de sostenibilidad o RSC no responden a un verdadero proceso de rendición de cuentas. Existe un fuerte debate en la academia sobre la utilidad y falta de credibilidad de los informes de sostenibilidad (Adams y Evans, 2004; Dando y Swift, 2003). Pese al crecimiento en el número de informes de sostenibilidad durante las dos últimas décadas, este fenómeno no ha venido acompañado por avance significativo en los contenidos ni en una involucración de las partes interesadas en el proceso (Gray, 2000; O’Dwyer y Owen, 2005).

Otro aspecto que dificulta el análisis desde una perspectiva de rendición de cuentas es que la RSC no es una actividad sistemática y al no existir una regulación en cuanto a la divulgación de información ésta puede variar en popularidad, en las materias que se presta mayor o menor atención y en qué tipo de organizaciones presentan esta información (Gray et el. 1995: 49). El argumento de Gray viene ratificado por el estudio "State of Sustainable (2014)"10, realizado por la empresa The Business for a Better World (2014), basado en una encuesta con una muestra de más de 700 personas mayoritariamente directivos de empresas (92\%) de todo el mundo que apunta el cambio en cuanto a las prioridades para los 12 meses siguientes en relación a las diferentes dimensiones de la RSC en el periodo 2009-2014. Por ejemplo, los derechos humanos se han convertido en la primera preocupación en 2014 mientras que en 2009 era la cuarta. Los derechos de los trabajadores de la tercera a la segunda y el cambio climático de la primera en 2009 a la tercera posición en 2014.

De acuerdo con el informe "measuring Sustainability Disclosure: Ranking the World's Stock Exchanges" elaborado por Corporate Knights Capital (2014) solamente 128 empresas de las 4.609 mayores empresas cotizadas en el mundo a diciembre de 2012, aquellas que tienen una capitaliza-

7.- www.accaglobal.com

8.- www.eurosif.org

9.- $h$ ttp://www.accaglobal.com/content/dam/acca/global/PDF-technical/sustainability-reporting/tech-tp-wdir.pdf

10.- $h$ ttp://www.bsr.org/reports/BSR_GlobeScan_Survey_2014.pdf 
ción por encima de 2 billones de dólares (escala corta utilizada en Estados Unidos), informan sobre los siete indicadores de primera generación: rotación de los empleados, energía, emisiones de gases de efecto invernadero (GEI), accidentes laborales, gastos salariales, residuos y agua. De acuerdo con este mismo estudio se ha producido un estancamiento en el avance de la información proporcionada por las empresas desde 2011. Para Milde y Gray (2012:17) los informes de sostenibilidad, salvo algunas pocas excepciones, incluyen a pocos grupos de interés y generalmente ignoran los principales problemas sociales que surgen de la actividad empresarial, como el lobby, la publicidad, el aumento del consumo, la distribución de la riqueza y así sucesivamente. Los informes a menudo se refieren a la "sostenibilidad" y "desarrollo sostenible", pero dejan cuestiones virtualmente sin respuesta como la huella ecológica, o la equidad y la justicia social.

La interpretación y utilidad de los informes de sostenibilidad afronta importantes retos, entre ellos la materialidad, la exhaustividad, comparabilidad y fiabilidad de la información. Muchos de estos retos vienen provocados porque la información no financiera permanece en el estadio de la voluntariedad en la mayor parte de los países, por la falta de armonización sobre el alcance de la información y la cobertura de temas y la carencia efectiva de sistemas de revisión o auditoria profundos e independientes.

\section{3.- Factores determinantes en la presentación de información no financiera}

No hay un acuerdo en la literatura sobre la teoría que soporta el que las empresas faciliten información ASG. Los estudios hasta la fecha se apoyan en diferentes perspectivas teóricas que van desde la teoría de la legitimidad, la teoría de los stakeholders hasta teorías políticas y económicas. Dentro de las teorías sobre las que se apoyan los estudios que evalúan la cantidad y calidad de información no financiera, Gray et al. (1995) distinguen entre teorías económicas y teorías políticas y sociales. Dentro de las primeras encontramos la teoría de agencia y la de la contabilidad positiva. Para Gray et al. (1995) estas últimas no son relevantes desde una perspectiva de RSC y lo justifica en que se basan en la existencia de un mercado "supuestamente libre" y esto va en contra de las principales preocupaciones de la RSC, que vienen motivadas básicamente por fallos y desajustes del mercado, y en el deseo de cambio de las prácticas actuales.

Hay diversos estudios que tratan de correlacionar los mecanismos de institucionalización (Jairo et al., 2013; Jennings y Zandbergen 1995; DiMaggio y Powell, 1983) apoyándose en la teoría de la legitimidad para explicar por qué las empresas presentan información social y medioambiental (Patten, 1992; Brown y Deegan, 1998). De acuerdo con la teoría de la legitimidad las empresas presentarían información para legitimarse ante la sociedad. La teoría de la legitimidad es definida por Suchman 
(1995: 574) como "una percepción generalizada o asunción de que las acciones de una entidad son deseables y adecuadas dentro de un sistema socialmente estructurado de normas, valores, creencias y definiciones".

Para O'Donovan (2002) la teoría de la legitimidad está basada en que la empresa para su subsistencia debe operar de acuerdo con lo que la sociedad considera un comportamiento socialmente responsable. En el ámbito de la comunicación, y según señalan Archel y Lazirraga (2001: 135), la política informativa de la empresa suele estar guiada por la necesidad de justificar ante la sociedad determinadas actuaciones empresariales. De acuerdo con la teoría de la legitimidad, las empresas divulgarán información en base a las demandas y preocupaciones sociales. Por ello se puede concluir que la presión social a la que se vea sometida la empresa va resultar determinante en la información social y medioambiental proporcionada por la misma

Jairo et al. (2013), a partir del estudio de la información proporcionada por empresas cotizadas de cinco países latinoamericanos, parten de diversas hipótesis para determinar qué factores influyen en una mayor información. Jairo et al. (2013) llegan a la conclusión de que la mayor respuesta de las empresas a proveer información extra-financiera se debe a mecanismos de institucionalización, entendidos estos como variables de contexto social que van desde reglas jurídicas y sociales, a los valores, usos y costumbres. En este sentido cobra especial relevancia la teoría de la legitimidad que explicaría un mayor nivel de información para justificar su comportamiento en base a un sistema de normas, valores y creencias vigentes en un momento determinado en la sociedad en la que está inmersa (Suchman, 1995). Para O'Donovan (2002) la teoría de la legitimidad está basada en que la empresa para su subsistencia debe operar de acuerdo con lo que la sociedad considera un comportamiento socialmente responsable.

Ullman (1985: 552) señala "Muchos investigadores en el área de gestión estratégica han subrayado la importancia de los valores y actitudes en el proceso de formulación de la estrategia (...) , más aún en el contexto de la respuesta a las demandas sociales". Diversos autores apoyan la tesis de que una mayor cultura social y medioambiental favorece más cantidad de información social y medioambiental en los informes anuales (Gray et al, 1995; Andrews et al., 1989). Moneva y Llena (2000) llegan a la conclusión a través de un estudio sobre la evolución de la información medioambiental en grandes empresas de que la evolución positiva está motivada por presiones sociales.

Para Campbell (2006) las empresas tendrán una mejor predisposición a un comportamiento socialmente responsable y por tanto a facilitar más información cuando se enfrentan a una regulación fuerte de los Estados, a presiones ejercidas desde las ONG y otros grupos organizados independientes, a la autorregulación por parte del sector o a la industria a la que pertenece la empresa. DiMaggio y Powell (1983) establecen tres isomorfismos dentro de los mecanismos de institucionalización que median entre el ámbito organizacional y el ámbito institucional: isomorfismo normativo, coercitivo y mimético. Este modelo es replicado para analizar los factores que inciden en la información ASG por Jennings y Zandbergen (1995), Jairo et al. (2015) y Amran y Haniffa (2011). Los mecanismos de ins- 
titucionalización son entendidos como variables de contexto social que van desde reglas jurídicas y sociales (formales e informales), valores, usos y costumbres (North, 1998).

DiMaggio y Powell (1991) definen el isomorfismo como el proceso por el que dos organizaciones diferentes adoptan una forma o comportamiento similar. El isomorfismo mimético es descrito por DiMaggio y Powell (1983) es aquel que es seguido por las compañías y sus directivos cuando contrastan el éxito de iniciativas innovadoras, en este caso de RSC, emprendidas desde otras empresas en un contexto de incertidumbre. El coercitivo es la respuesta por parte de la empresa a la presión ejercida por parte de los actores que están en su entorno.

Los aspectos de responsabilidad social han sido ampliamente estudiados por la academia y también los factores que han contribuido a que las empresas presenten información extra-financiera. Hay diversos estudios longitudinales que tratan de valorar la evolución en la cantidad y calidad de información suministrada por la empresa en materia social y medioambiental, e identificar los factores que influyen en ambas variables, cantidad y calidad (Deegan y Gordon, 1996; Gray et al., 1995; Brown y Deegan, 1998; Moneva y Llena, 2000, y Archel, 2003, Jairo et al., 2013). Pese a la cantidad de estudios existentes, no hay pruebas consistentes de qué factor es verdaderamente determinante en el ámbito de la provisión e información no financiera por parte de la empresa (Orij, 2010). Esta falta de acuerdo se debe a que los estudios emplean diferentes modelos, tratan de dar respuesta a distintas cuestiones y el alcance es diverso tanto desde una perspectiva de países como de periodos analizados. Los estudios hasta la fecha se apoyan en diversas perspectivas teóricas que van desde la teoría de la legitimidad, la teoría de los stakeholders hasta teorías políticas y económicas.

Por otro lado hay escasos estudios que tengan un alcance de varios ejercicios con el objetivo de valorar los factores institucionales a través del análisis de un número de empresas en un país o región determinada, y que aborden todos los aspectos de responsabilidad social en el análisis. La mayor parte de los estudios se constriñen al análisis de información medioambiental e incluso en estos trabajos el peso e importancia en el análisis de temas y aspectos concretos difiere en base al origen geográfico tanto del investigador como de las empresas analizadas. Por ejemplo en Estados Unidos se le da mayor importancia a los temas relacionados con producto y energía que en terceros países (Hackston y Milne, 1996).

En España, los estudios existentes se han limitado al análisis de aspectos concretos de la información no financiera no abarcando todas las dimensiones de la RSC. Tal es el caso de Archel y Lizarraga (2001) Archel (2003), Moneva y Llena (2000), Larrinaga et al. (2002) que evalúan los factores determinantes en la información medioambiental. Moneva y Llena (1996) extienden el análisis a la información sobre personal y sobre la labor social de la empresa aunque con un alcance muy limitado en cuanto a los aspectos a analizar. Ponce y Labat (2011) se limitan al análisis de la información social.

Parte de la literatura revisada encuentra una correlación entre el país en el que se emite la información y el país de origen de la propiedad de la empresa y la calidad y cantidad de información social y medioambiental facilitada por la empresa (Andrews et a, 1989; Teoh y Thong, 1984). 
Por otro lado, hay países que han incluido requerimientos específicos de carácter obligatorio en materia de información social y medioambiental. Dinamarca y Francia son dos de los países y de acuerdo con KPMG y GRI (2013) los resultados son positivos en términos de cantidad de empresas que informan y calidad de los informes. Además, las presiones sociales no solamente pueden provocar una mayor información por parte de la empresa sino también una actitud más proclive a regular por parte de los Estados aquellos aspectos relativos a la información de carácter social y medioambiental. Este factor, el regulatorio, como hecho o como evidencia de que se producirá en un futuro cercano, puede ser un elemento clave en la cantidad y calidad de información presentada por la empresa. De acuerdo con Idowu y Papasolomou (2007), entre las diversas razones de la empresa para presentar información no financiera está el factor de cumplimiento con los requerimientos de los gobiernos. The KPMG Survey of Corporate Responsibility Reporting 2013 (KPMG, 2014) realizado sobre una muestra de 4.100 empresas en todo el mundo concluye que los requerimientos regulatorios han conducido a los más altos niveles de información a Francia, Dinamarca o Sudáfrica en comparación con otros países.

Otros investigadores relacionan la cantidad y calidad de la información con características propias de la empresa (capitalización, beneficios, industria a la que pertenecen, país origen de la propiedad, cualidades de los directivos, procesos de gestión, entre otros). En relación al tamaño de la empresa hay diversos estudios que establecen una correlación positiva entre esta variable y la información social y medioambiental facilitada (Cowen et al., 1987; Kelly, 1981; Pang, 1982; Patten, 1991, 1992) aunque tal y como apuntan Gray et al. no parece determinante si no se tienen en cuenta otros factores (Gray et al. 1995: 49).

El resultado de la investigación que se presenta a continuación tiene como objetivo evaluar los factores que son determinantes en la presentación de información extra financiera por parte de las empresas que componen el selectivo español lbex 35 tomando como base la teoría de la legitimidad.

\section{4.- Análisis de la evolución de la calidad de la información en las empresas del IBEX 35}

La presente investigación tiene como objetivo principal aportar una mayor compresión de los factores que determinan que las empresas españolas, más concretamente las incluidas en el selectivo español Ibex 35, presenten información no financiera. Para ello se establecen diversas hipótesis de partida que vendrían a corroborar que las empresas presentan información con carácter voluntario y no financiera para legitimarse ante la sociedad. 
H1: Las empresas que pertenecen a sectores expuestos a mayores riesgos sociales y medioambientales facilitan más información ASG y de mejor calidad.

H2: Las presiones de la sociedad civil son un factor determinante en la información suministrada por la empresa.

H3: El efecto mimético es un factor determinante en la calidad de la información social y medioambiental.

H4: Los aspectos sobre los que más información provee la empresa son aquellos en los que existe una regulación o bien una mayor exigencia en cuanto a la información a suministrar por parte de los poderes públicos.

\subsection{Metodología}

En el estudio se ha determinado para su evaluación un período de informes anuales de 7 años, que comprende las memorias publicadas en los años 2005-2011 por las empresas del lbex 35, correspondientes a los informes realizados por el Observatorio de RSC en los años 2007-2013 ${ }^{11}$.

Con objeto de hacer el estudio lo más homogéneo posible, se ha seleccionado una muestra de 26 empresas que han permanecido en el IBEX 35 en el período seleccionado un mínimo de 6 años, 0 bien estaban en el selectivo y posteriormente han pasado a formar parte de otra de las empresas presentes en el lbex 35, ya sea por fusión o absorción. Estas empresas se presentan en el cuadro 1:

\section{Cuadro 1. Listado de empresas de la muestra}

\begin{tabular}{|ll|}
\hline Abertis & Acciona \\
Acerinox & ACS \\
Banesto & Bankinter \\
BBVA & Banco Popular \\
Banco Sabadell & Enagás \\
Endesa & FCC \\
Ferrovial & Gamesa \\
Gas Natural & Iberdrola \\
IAG Iberia & Inditex \\
Indra & Mapfre \\
REE & Repsol \\
SCH & Telecinco \\
Telefónica & Unión Fenosa \\
\hline
\end{tabular}

FUENTE: Elaboración propia.

11.- El modelo de indicadores analizados por el Observatorio de Responsabilidad Social Corporativa se basa en cinco normas o códigos de amplio reconocimiento y aceptación internacional: La versión 2 y 3 de Global Reporting Initiative (GRI), el modelo de aseguramiento y los principios establecidos en el mismo por la New Economics Foundation (NEF), la norma AA 1000 de AccountAbility, las normas de Naciones Unidas para empresas multinacionales y otras empresas comerciales y códigos de gobierno corporativo (Aldama, Olivenza, Código Conthe y Ley Sarbanes Oxley). 
De estas 26 empresas, 23 de ellas aparecen todos los años del estudio. Mapfre no aparece en el 2005 ya que no estaba incluida en el IBEX; Unión Fenosa y Gas natural desaparecen en el 2009 debido a su fusión, convirtiéndose ese año en Gas Natural Fenosa; así mismo, Banesto, que no estaba en el selectivo el año 2005, se integró en el Grupo Santander en el año 2011.

El Observatorio de RSC evalúa para cada empresa una serie de indicadores cuantitativos, estableciendo valoraciones entre 0-4 en función de la calidad y cantidad de la información presentada. Además de estos indicadores cuantitativos en el modelo de análisis seguido por el Observatorio de RSC se tienen en cuenta una serie de indicadores cualitativos. Son indicadores que verifican el cumplimiento de los aspectos analizados como, por ejemplo, si tienen una política medioambiental. Para la realización de la presente investigación se ha extraído la información de la base de datos del Observatorio, tomando como base para el análisis longitudinal los indicadores de tipo cualitativos que hayan permanecido durante todo el período analizado (2005-2011) y dejando para una posterior investigación el análisis de los indicadores cuantitativos. Los indicadores cualitativos analizan si la empresa presenta información en las dimensiones de medio ambiente, económica, derechos laborales, corrupción y políticas públicas, derechos humanos, consumo y gobierno corporativo. Una vez recopilados los datos se ha procedido a hacer una revisión de los indicadores por empresa en todos los ejercicios objeto de análisis (2005-2011) corrigiendo las incoherencias o errores identificados.

Los estudios realizados por el Observatorio de RSC han sido previamente utilizados por otros investigadores como el caso de Reverte $(2009,2012)$ para evaluar la evolución de la información. La diferencia con la presente investigación estriba en el periodo temporal abarcado, en el caso de Reverte en un primer estudio evalúa la progresión de la información en el periodo 2005 al 2006 y en un segundo estudio evalúa la evolución desde el 2003 al 2008. Ambos estudios elaborados por Reverte se basan en las valoraciones e indicadores cuantitativos obtenidos por el Observatorio de RSC, aspecto no susceptible de revisión por el autor al no tener acceso a la herramienta utilizada por el observatorio de RSC y por tanto desconocer el contenido de los indicadores utilizados por esta institución. Además, Reverte no distingue la evolución por dimensiones sino que incorpora evaluaciones globales. 


\begin{tabular}{|c|c|c|c|c|c|c|c|c|c|c|}
\hline & 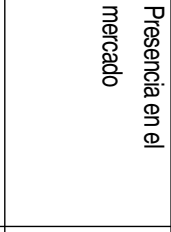 & 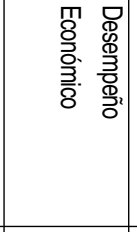 & 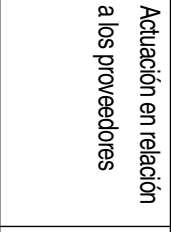 & 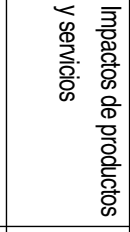 & 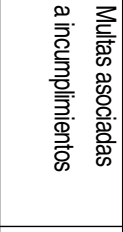 & 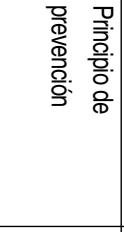 & & 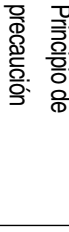 & 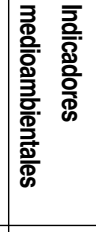 & 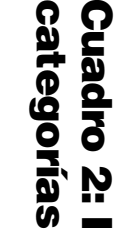 \\
\hline & & 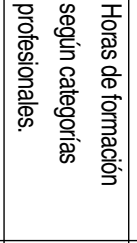 & 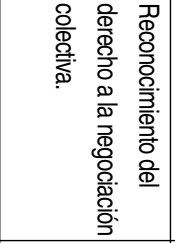 & 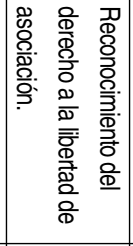 & 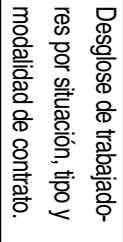 & 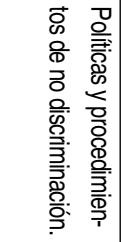 & 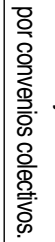 & 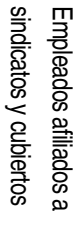 & 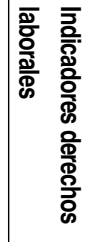 & $\begin{array}{l}\frac{2}{8} \\
\frac{1}{2} \\
\frac{0}{0} \\
\frac{1}{8}\end{array}$ \\
\hline & 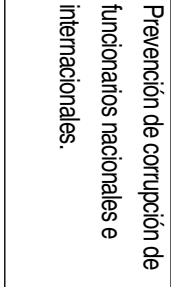 & 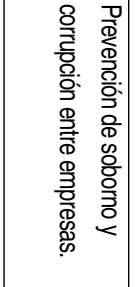 & 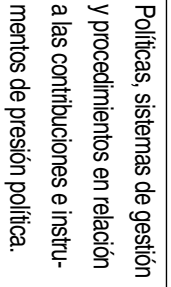 & 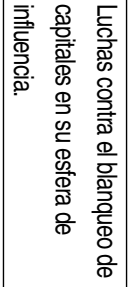 & 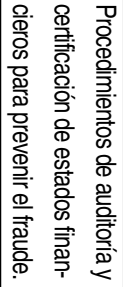 & 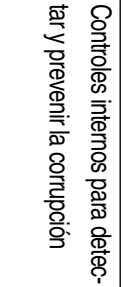 & $\begin{array}{l}\text { o } \\
\text { 亮 } \\
\text { 兽: }\end{array}$ & 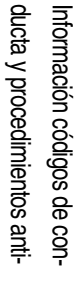 & 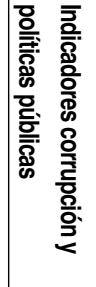 & 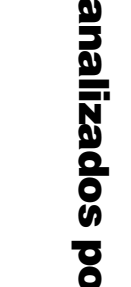 \\
\hline 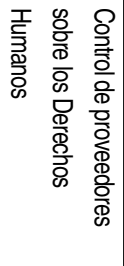 & 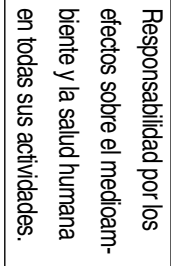 & 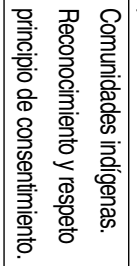 & 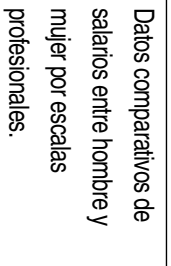 & 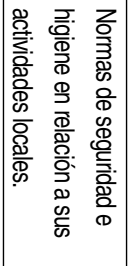 & 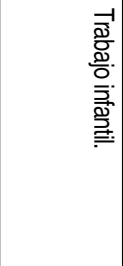 & 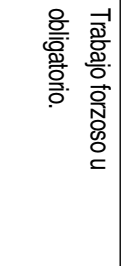 & 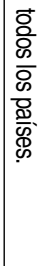 & 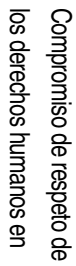 & 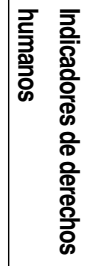 & 8 \\
\hline & 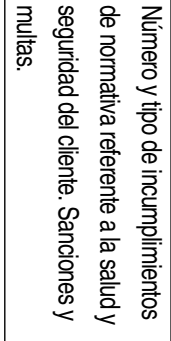 & 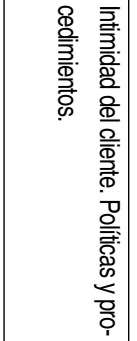 & 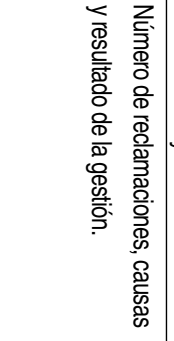 & 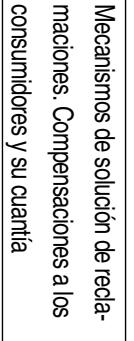 & 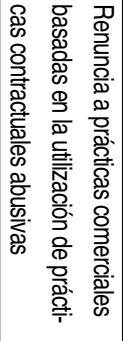 & 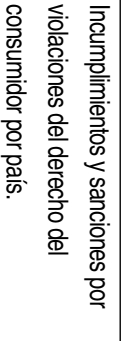 & & 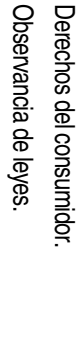 & 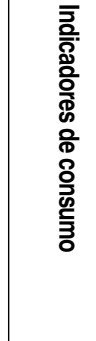 & 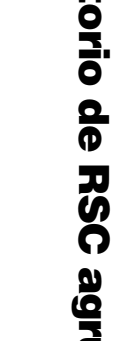 \\
\hline 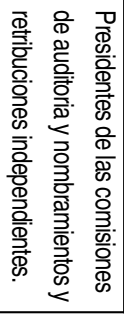 & 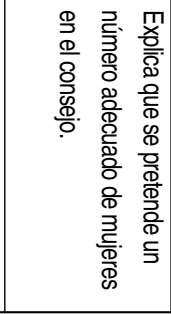 & 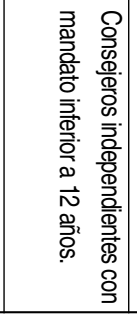 & 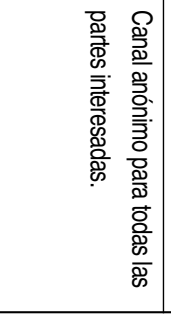 & 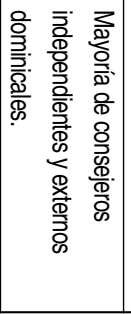 & 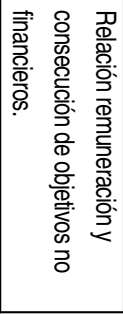 & 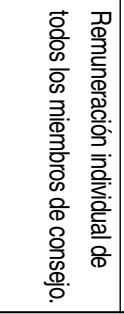 & & 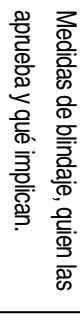 & 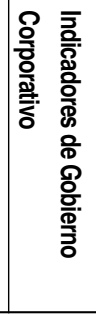 & 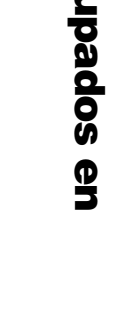 \\
\hline
\end{tabular}




\subsection{Principales resultados del estudio}

En este apartado se realiza una agregación de todas las dimensiones analizadas y se extraen las medias de cada sector para todos los indicadores, obteniendo un porcentaje global de resultados en todos los ámbitos estudiados.

El sector que mejor puntuación global alcanza a diciembre de 2011 es petróleo y energía. Este sector representado por Enagás, Endesa, Gamesa, Gas Natural Fenosa, Iberdrola, Red Eléctrica y Repsol obtiene un nivel de cumplimiento del $49,14 \%$ del total de indicadores cualitativos analizados, lo que indica que las empresas del sector informan correctamente de casi la mitad de los indicadores propuestos en todas las dimensiones analizadas. El avance global por parte del sector de petróleo y energía es significativo en los 7 años objeto de análisis pasando de un $20 \%$ de cumplimiento en el año 2005 a un 49,14\% en 2011. Sus buenos resultados están basados en las dimensiones de derechos laborales, corrupción, derechos humanos y gobierno corporativo donde superan el $50 \%$ e incluso el $60 \%$ en el caso de Gobierno Corporativo.

\section{Gráfico 3. Evolución de los indicadores agregados por sectores}

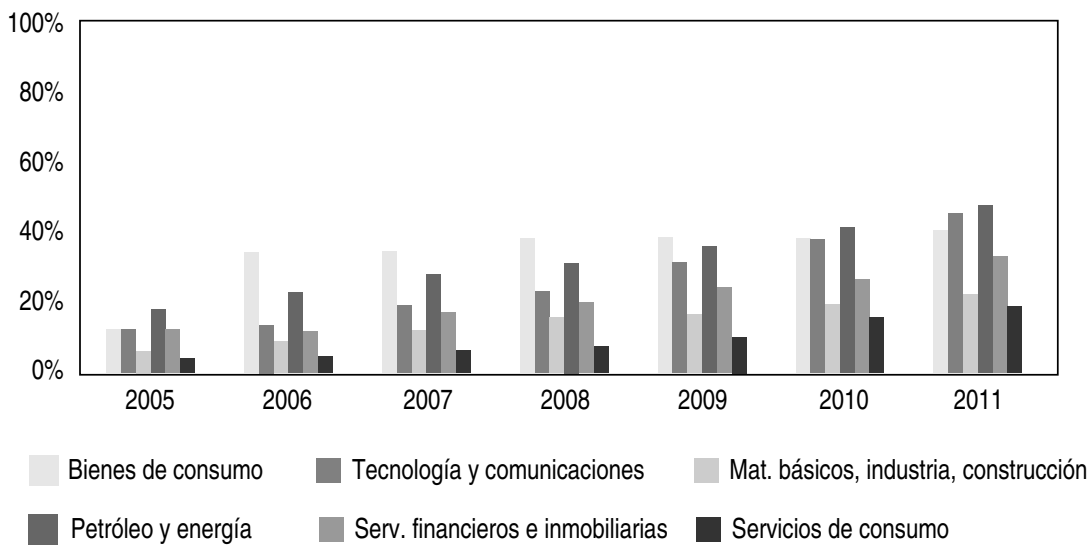

FUENTE: Elaboración propia a partir de los datos obtenidos del Observatorio de Responsabilidad Social Corporativa.

H1: Las empresas que pertenecen a sectores expuestos a mayores riesgos sociales y medioambientales facilitan más información ASG y de mejor calidad 
El argumento de que aquellos sectores con mayores impactos sociales y medioambientales recaban mayor atención por parte de los medios de comunicación y se encuentran sometidos a mayores presiones sociales lo que desemboca en que faciliten más información y de mayor calidad ha sido ampliamente apoyados por parte de la literatura. Roberts (1992), Deegan y Gordon (1996) llegan a la conclusión de que el tipo de actividad desarrollado por la compañía es un factor determinante en la presentación de la información. Aquellas empresas que pertenecen a sectores de industrias contaminantes tienen que hacer frente a una mayor regulación, a una mayor vigilancia por parte de medios de comunicación y a una mayor presión de diversos grupos de interés (Deegan y Gordon, 1996).

Liu y Ambumozhi (2009) a través de una investigación sobre la información medioambiental en 175 empresas chinas cotizadas señalan la exposición y sensibilidad medioambiental de las compañías como el factor más determinante, junto con el tamaño, en la calidad de la información suministrada.

Hackston y Milne (1996) y Archell (2003) consideran empresas críticas en materia medioambiental a las pertenecientes a los sectores de energía, petróleo, química y metal y automóvil.

De acuerdo con Jean Founder, CEO de Sustainability Accounting Standards Board, los sectores del petróleo, gas y minería son más transparentes que otros por estar sometidos a un mayor escrutinio tanto social como por parte de los inversores, por tener altas tasas de siniestralidad laboral y por ser sectores forzados a la innovación debido a los riesgos a los que están expuestos ${ }^{12}$.

Patten (1992) concluye que las compañías petroleras presentan más información medioambiental a partir del accidente del Exxon Valdez al verse sometidas a mayores presiones tanto sociales como por parte del regulador.

Moneva y Llena (2000) a través de un estudio sobre la evolución de la información medioambiental en grandes empresas españolas concluyen que la evolución positiva en ésta se debe a presiones sociales. Archel (2003) a través de la creación de un índice general de evaluación para el período 19941998 para las dimensiones social, ambiental y laboral tomando como muestra a 62 grandes empresas españolas concluye que las empresas eléctricas junto con las de petróleo son las que mayor información y mejor evolución presentan lo que puede interpretarse como fruto de la presión ejercida desde grupos sociales hacia aquellos sectores que tienen mayores impactos en los ámbitos social y medioambiental. Considera la pertenencia a un sector crítico como variable explicativa de la divulgación de información social y medioambiental. A los mimos resultados llegan Gómez y Laburu (2008) que concluyen a través de un análisis de contenido de las dimensiones de empleados y medioambiente de las empresas incluidas en los índices bursátiles lbex 35 y el Dax 30 que las empresas del sector petró-

12.- Rogers, J. (2013). 4 signs of sustainability from oil, gas and mining companies [Web log comentario]. Extraído el 14 de Agosto de 2015 de http://www.greenbiz.com/blog/2013/11/05/4-sustainability-trends-oil-gas-mining. 
leo y energía son las que más información y de mejor calidad proporcionan y que esto podría responder a la necesidad de las empresas del sector de lograr su legitimidad social. Reverte (2009) por su parte concluye que en las empresas del lbex 35 el factor determinante en la presentación de información ASG es, en primer lugar, el grado de exposición pública de la empresa, seguida por el tamaño y la industria a la que pertenece. Concluye que debido a los resultados parece que la teoría de la legitimidad es lo que mueve a las empresas españolas a informar sobre aspectos ASG.

Ponce y Labat (2011) a través de una investigación que toma como muestra a las empresas del Ibex 35 durante el periodo de 1996 a 1999, concluyen que el sector de actividad es, junto con el tamaño, la variable explicativa de la relevación de información de carácter social.

Prakash (2015) analiza la calidad de la información en a 614 grandes empresas cotizadas de 43 países pertenecientes a 20 industrias diferentes y concluye que los sectores de Minería petróleo y gas son los que mejor valoración obtienen seguidos de la industria automovilística y telecomunicaciones.

\section{H2: Las presiones de la sociedad civil son un factor determinante en la información sumi- nistrada por la empresa.}

La mayor parte de las empresas energéticas incluidas en el Ibex 35 han sido objeto de diversas campañas y denuncias por los impactos y externalidades provocadas en el medioambiente, sobre los derechos humanos y sobre el consumidor.

Repsol acumula, durante el periodo analizado (2005-2011), un gran número de denuncias por violaciones a los derechos humanos, daños ambientales irreversibles e importantes impactos sociales, así como perjuicios económicos en Argentina, Bolivia, Colombia, Ecuador, Perú y España.

Merece especial atención por los efectos obtenidos en la información proporcionada por Repsol la campaña que realizó la ONG Oxfam Intermon en favor de los pueblos indígenas y de la publicación de información financiera país a país en 2009. La campaña concluye con una acción de activismo accionarial en la Junta de Accionistas de Repsol celebrada en Madrid el 14 de mayo de 2009. La ONG consiguió la cesión de derechos políticos de accionistas particulares y gestoras de fondos de inversión de España, Estados Unidos y Noruega. La respuesta del presidente de la empresa, Antonio Brufau, en la Junta General de Accionistas a la demanda planteada por Repsol fue altamente positiva: "...estoy totalmente de acuerdo con Uds en los dos campos. En lo que es la colaboración con la información pública y en lo que es la involucración con todo nuestro entorno donde operamos...Me pongo a su disposición y estoy enteramente a lo que Uds. digan pues para intercambiar aquellos puntos de vista para mejorar nuestra transparencia en la información...y estudiar todos los proyectos que supongan un mejor entorno de vida de las comunidades indígenas en donde estamos nosotros"13. 2J6_T7A.

13.- Texto extraído de vídeo de canal youtube de Oxfam Intermon el 01 de septiembre de 2015: https://www.youtube.com/watch?v=e_v- 
Repsol en 2010 aprueba una política que reconoce los derechos de los pueblos indígenas y publica en su memoria de 2010 los mecanismos implantados. Repsol, se convierte así en la única empresa del Ibex 35 que informa sobre su política de relación con comunidades indígenas y que reconoce expresamente el respeto al principio de consentimiento de los pueblos y comunidades indígenas a ser afectados por sus proyectos de desarrollo.

Endesa acumula durante el periodo analizado (2005-2011), un gran número de denuncias por violaciones a los derechos humanos, daños ambientales irreversibles e importantes impactos sociales, así como perjuicios económicos en Argentina, Brasil, Chile, Colombia, Guatemala, Perú y España (ver informe elaborado por el Observatorio de RSC para el observatorio de publicidad) ${ }^{14}$.

Iberdrola acumula durante el periodo analizado (2005-2011), un gran número de denuncias por violaciones a los derechos humanos, daños ambientales irreversibles e importantes impactos sociales, así como perjuicios económicos en México, Brasil, Chile, Bolivia, Guatemala y España (ver informe elaborado por el Observatorio de RSC para el observatorio de publicidad, 2011 ${ }^{15}$ )

Unión Fenosa acumula durante el periodo analizado (2005-2011), un gran número de denuncias por violaciones a los derechos humanos, daños ambientales irreversibles e importantes impactos sociales, así como perjuicios económicos en Panamá, Nicaragua, Colombia, Guatemala, Costa Rica y España (ver informe elaborado por el Observatorio de RSC para el observatorio de publicidad ${ }^{16}$ ). Unión Fenosa ha sido objeto de campañas en España y en distintos países de América Latina. La campaña que más repercusión ha tenido es la de la Ir-responsabilidad social de Unión Penosa en la que han participado más de 50 organizaciones y realizado acciones de denuncia, publicación de artículos en prensa, cartas y correos electrónicos dirigidos a Gobiernos, a accionistas de la empresa y a la propia empresa. El caso de Unión Fenosa ha sido objeto de diversas publicaciones, entre ellas el libro "La energía que apaga Colombia: Los impactos de las inversiones de Repsol y Unión Fenosa" (Ramiro et al, 2007).

El tercer sector que obtiene la nota más alta es el de bienes de consumo, representado por Inditex, con un $42 \%$ de nivel de cumplimiento sobre los indicadores analizados a 31 de diciembre de 2015. Sin embargo, la falta de más empresas en la muestra dificulta el examen de dicho sector, que fundamenta sus buenos resultados de información en las dimensiones de derechos laborales, corrupción y derechos humanos, superando en todas ellas el $60 \%$ de cumplimiento de los indicadores. Inditex se encuentra muy posicionada en las dimensiones de Derechos Laborales y Derechos Humanos, uno de los factores fundamentales es el elevado riesgo del sector a la vulneración de los derechos humanos en su cadena de producción y las presiones sociales recibidas por parte de sindicatos y ONG, entre ellas

14.- http://www.fundacion-ipade.org/observatorio-publicidad/wp-content/uploads/2011/06/Informe-ENDESA.pdf.

15. - $h$ ttp://www.fundacion-ipade.org/observatorio-publicidad/wp-content/uploads/2011/08/Informe-lberdrola.pdf.

16. - http://www.fundacion-ipade.org/observatorio-publicidad/wp-content/uploads/2011/08/Informe-UNION-FENOSA.pdf. 
la campaña Ropa Limpia. Inditex fue la primera empresa española que fue objeto de una acción de activismo accionarial para reclamar un mejor control de su cadena de producción.

\section{H3: El efecto mimético es un factor determinante en la calidad de la información social y medioambiental presentada por las empresas.}

DiMaggio y Powell (1983) establecen que existen mecanismos de institucionalización denominados isomorfismos que explican que las empresas reproduzcan o asuman comportamientos similares en un campo institucional. En este sentido, subrayan tres formas de isomorfismos: el coercitivo, el normativo y el mimético. Jairo et al., (2013) llegan a la conclusión después de analizar la divulgación de información sobre RSC de las empresas cotizadas de los países de Latinoamérica con costa en el Océano Pacífico que las empresas tienden a emular el comportamiento de los líderes de su sector. El isomorfismo mimético explicaría que las empresas del sector energético sean las que más información presentan en las dimensiones analizadas en el presente estudio. Las empresas incluidas en estos sectores se encuentran entre las primeras en el ranking que elabora anualmente el Observatorio de RSC. Lo anterior genera un efecto mimético en las compañías del sector por intentar posicionarse en línea con la competencia directa. En el Índice de Sostenibilidad de 2011 que elabora SAM ${ }^{17}$ (2011) el sector energético y el gasístico se encuentra en las primeras posiciones con respecto a otros sectores. Las empresas del sector eléctrico, del petróleo y gas que cotizan en el selectivo español lbex 35 se encuentran altamente internacionalizadas y por tanto compiten en mercados globales donde los aspectos ligados a la sostenibilidad del negocio son cada vez más tenidos en consideración.

En segundo lugar se sitúa el sector de tecnología y comunicaciones con un $47 \%$ de cumplimiento de los indicadores analizados a diciembre de 2011. En este sector sólo están presentes Telefónica e Indra, lo que dificulta la extrapolación de los datos. Como en el caso del sector energético y gasístico el de telecomunicaciones ocupa posiciones altas en el Índice de Sostenibilidad de SAM. El resultado también es coincidente con el estudio elaborado por Prakash (2015) que analiza la calidad de la información en 614 grandes empresas cotizadas de 43 países pertenecientes a 20 industrias diferentes y concluye que los sectores de Minería petróleo y gas son los que mejor valoración obtienen seguidos de la industria automovilística y telecomunicaciones. La posición puede justificarse por el nivel de competitividad existente en el sector de las comunicaciones y el consiguiente efecto mimético que las empresas que operan en un entorno internacional y cotizan en diversos mercados llevan a cabo para posicionarse en los primeros puestos. Telefónica, una de las dos empresas que integran el sector, está altamente internacionalizada con presencia en más de 15 países, sus acciones cotizan en varios mercados (Madrid, Lima, Buenos Aires y New York) y están incluidas en más de 100 índices ${ }^{18}$. Es destacable mencionar la progresión llevada a cabo por este sector, que durante los 7

17.- Entidad que analiza el desempeño económico, ambiental y social de las principales corporaciones del mundo en su "The Sustainability Yearbook".

18.- Más información en http://www.telefonica.com/es/shareholders-investors/jsp/invertia/sector.jsp. 
años analizados ha conseguido la mayor mejora en la calidad de la información pasando del 14\% al $47 \%$ de indicadores informados correctamente.

Por otro lado, las presiones sociales no pueden provocar una mayor información por parte de la empresa sino también una actitud más proclive a regular por parte de los Estados los aspectos relativos a la información de carácter social y medioambiental.

H4: Los aspectos sobre los que más información provee la empresa son aquellos en los que existe una regulación o bien una mayor exigencia en cuanto a la información a suministrar por parte de los poderes públicos.

Las empresas pertenecientes al sector de petróleo y energía se han visto sujetas a una creciente legislación, básicamente en materia medioambiental, que directa o indirectamente puede haber afectado a que se provea de más información y de mayor calidad por parte de las empresas incluidas en el sector de petróleo y energía. Estos marcos regulatorios se ha concretado en la Ley 54/1997, de 27 de noviembre, del Sector Eléctrico (LSE, 1997) que establece en su artículo 20.2 para las empresas eléctricas la inclusión en las cuentas anuales de la información relativa a las actuaciones empresariales con incidencia sobre el medio ambiente; la Directiva 2001/42/CE, relativa a la evaluación de los efectos de determinados "Planes y Programas en el Medio Ambiente", exige que el órgano de la Administración que sea promotor de un Plan o Programa, elabore un "Informe de Sostenibilidad Medioambiental"; la "Ley sobre evaluación de los efectos de determinados planes y programas en el medio ambiente", conocida como Ley 9/2006; la Directiva 2004/35/CE, sobre "Responsabilidad Medioambiental en relación con la prevención y reparación de daños medioambientales", norma modificada por la Directiva 2006/21/CE, sobre la gestión de los residuos de industrias extractivas; la Directiva 2003/87/CE, que contempla un régimen para el comercio de derechos de emisión de gases de efecto invernadero en la Comunidad. Además, dentro del "Programa Europeo de Cambio Climático", constituye la iniciativa más relevante de la Unión Europea para lograr que la Comunidad y sus Estados miembros puedan cumplir el compromiso de reducción de emisión de gases de efecto invernadero dentro del Marco del Protocolo de Kioto. En este sentido, en España se ha publicado la Ley $1 / 2005$, de 9 de marzo, por la que se regula el régimen del comercio de derechos de emisión de gases de efecto invernadero.

Por otro lado, en relación a la evolución de la información por dimensiones e indicadores analizados presenta diferencias en cuanto a su nivel de avance en el periodo objeto de estudio (2005-2011). Idowu y Papasolomou (2007) presentan como resultados de un estudio que las empresas del Reino Unido tienen diferentes motivaciones para incluir información de RSC, entre las que se encuentran los requerimientos del Gobierno sobre determinados aspectos de RSC. Son varios los ejemplos de indicadores que experimentan una evolución positiva y que la misma puede atribuirse al efecto regulatorio o normativo: 
- En relación a la información sobre el principio de prevención hay un salto importante en 2008 , pasan de $58 \%$ a un $92 \%$ de cumplimiento sobre este indicador, que puede atribuirse a la inclusión del principio de prevención en la Ley 26/2007, de 23 de octubre, de Responsabilidad Medioambiental (LRM, 2007).

- En el ámbito laboral, los indicadores referidos a evitar discriminaciones y el abuso físico y verbal en el trabajo han tenido una evolución importante, el primero pasa de ser cumplido por un $8 \%$ de las empresas analizadas a más de un $50 \%$ en 2011 , y el segundo pasa de un $37 \%$ a más de un $83 \%$ de cumplimiento por parte de las empresas analizadas. Los resultados por tanto son muy positivos. En la evolución han podido influir los planes de igualdad elaborados por las empresas a partir de la Ley Orgánica 3/2007 para la igualdad efectiva de hombres y mujeres (LOIHM, 2007) y a la aprobación en 2009 del proyecto de Ley de Reforma de Código Penal (LOLOCP, 2010) que establece como hecho punible el acoso laboral a través de la modificación del artículo 172.

- En relación a corrupción y el indicador "políticas de lucha contra el blanqueo de dinero en su esfera de actividad" apenas ha tenido evolución, ya que ha pasado de un $20 \%$ a un $33 \%$ de cumplimiento. En este indicador el sector financiero vuelve a destacar significativamente teniendo la mayoría de las empresas que proveen esta información en sus memorias. Esto puede tener su explicación en la regulación existente en España en esta materia, primero con la Ley 12/2003, de 21 de mayo, de bloqueo de la financiación del terrorismo (LPBFT, 2003) y después con Ley 10/2010, de 28 de abril, de prevención del blanqueo de capitales y de la financiación del terrorismo (LOLOCP, 2010). A 31 de diciembre de 2011 todas las empresas del sector financiero incluidas en el análisis cumplen con este indicador.

- La nota diferencial en cuanto a las dimensiones analizadas la obtiene Gobierno Corporativo, cuyo nivel de cumplimiento global sobre todos los indicadores analizados en esta dimensión es del 52\%. Lo anterior es atribuible a la obligación establecida por el artículo 116 de la Ley 26/2003 del 17 de julio para las empresas cotizadas y otras empresas que emitan valores sujetos a negociación en los mercados financieros organizados a publicar anualmente informes de Buen Gobierno de acuerdo con unas recomendaciones que han ido variando con el tiempo. 


\section{5.- Conclusiones}

La información en el ámbito social, medioambiental y de buen gobierno ha evolucionado positivamente en los últimos 15 años, sin embargo todavía dista de responder a un proceso de rendición de cuentas. En cuanto a los factores que influyen en que las empresas presenten más o menos información y de mayor o menor calidad no hay uno que por sí solo sea determinante, sino que es producto de la conjunción de varios factores, entre los que destaca los elementos de carácter regulatorio y la presión social ejercida por ciudadanos y ONG a través de sus campañas. Tal y como señala Campdell (2006), las empresas se comportan de manera más socialmente responsable y por extensión facilitarán más información cuando se enfrentan a una regulación fuerte, códigos sectoriales de autorregulación, y presiones de organizaciones independientes como ONGs.

Las empresas analizadas tienden a informar sobre los impactos positivos de su actividad y omiten los negativos, lo que provoca el no cumplimiento de algunos indicadores como es el caso del impacto ambiental de productos o los relacionados con multas y sanciones.

En relación a los sectores, del estudio realizado se puede concluir que son aquellos con un mayor riesgo de impacto social y medioambiental los que atraen una mayor atención por parte del público y de los medios de comunicación sobre cómo gestionan las externalidades en el ámbito social y medioambiental. Este sería el factor determinante en que faciliten más información sobre cómo gestionan esos potenciales impactos con el objetivo de buscar una legitimidad ante las sociedades en las que operan. Por otro lado, un factor que puede motivar que se facilite más información es el nivel de internacionalización al tener que competir en mercados globales donde los aspectos ligados a la sostenibilidad del negocio son cada vez más tenidos en consideración. Las empresas tenderán a imitar a sus competidores en los compromisos en relación a la sostenibilidad y la información de carácter no financiero que facilitan al mercado.

Los resultados obtenidos en la presente investigación vienen a corroborar que las empresas facilitan información para legitimarse ante la sociedad y que los mecanismos de institucionalización relacionados con el entorno afectan a la cantidad y calidad de información suministrada. La empresa necesita adaptar sus valores y comportamientos a las expectativas de la sociedad y de los grupos de presión que actúan en el entorno, bien sean reguladores, consumidores u ONGs. La presión ejercida desde ONG, sindicatos y grupos de consumidores puede afectar directamente a cambios en el comportamiento y conducta de la empresa y a la información de carácter no financiero que facilita, y también puede influir en que los poderes públicos tiendan a regular ciertos aspectos, algunos de ellos relacionados con la transparencia. Las empresas tienden a informar con mayor detalle de aquellos aspectos que han sido objeto de una regulación previa o como en el caso de Buen Gobierno existe 
una normativa que obliga a las empresas a emitir un informe de gobierno corporativo conforme un modelo estandarizado.

Este estudio es el primero de una secuencia y por tanto tiene limitaciones derivadas de los indicadores analizados. Solamente se han tenido en cuenta los cualitativos, y los factores que puedan influir en la calidad y cantidad de información no financiera. Se deja para posteriores estudios el análisis de factores como el nivel de capitalización, los beneficios o cómo influyen los procesos organizativos en la información no financiera facilitada por la empresa.

\section{6.- Bibliografía}

ACCA \& EUROSIF (2013): "What do investors expect from non-financial reporting?", Disponible en: http://www.accaglobal.com/content/dam/acca/global/PDF-technical/sustainability-reporting/techtp-wdir.pdf.

ADAMS, C.A. \& EVANS, R (2004): "Accountability, completeness, credibility and the audit expectations gap", Journal of Corporate Citizenship, 14, 97-115.

AMRAN, A. \& HANIFFA, R. (2011): "Evidence in development of sustainability reporting: a case of a developing country", Business Strategy and the Environment, 20(3), 141-156.

ANDREWS, B., GUL, F., GUTHRIE, J. \& TEOH, H. (1989): "A note on corporate social disclosure practices in developing countries: the case of Malaysia and Singapore", British Accounting Review, 21 (4), 371-6.

ARCHEL, P. (2003): "La divulgación de la información social y medioambiental de la gran empresa española en el período 1994-1998. Situación Actual y perspectivas", Revista española de financiación y contabilidad, 117, 571-599.

ARCHEL, P. \& LIZARRAGA, F. (2001): "Algunos determinantes de la información medioambiental divulgada por las empresas españolas cotizadas", Revista de contabilidad, 4(7), 129-153.

BAILEY, D., HARTE, G. \& SUGDEN, R. (2000): "Corporate disclosure and the de-regulation of international investment", Accounting, Auditing and Accountability Journal, 13 (2), 197-218.

BLOOMBERG (2015): "Impact Report 2014". Disponible en: http://www.bloomberg.com/bcause/content/uploads/sites/6/2015/06/15_0608-Impact-Report_Web.pdf. 
BOSTON COLLEGE CENTER FOR CORPORATE CITIZENSHIP Y BUILDING A BETTER WORLD (2014): "Value of sustainability reporting In the 2013". Disponible en: http://www.ey.com/US/en/Services/Specialty-Services/Climate-Change-and-SustainabilityServices/Value-of-sustainability-reporting.

BROWN, N. \& DEEGAN, C. (1998): "The Public Disclosure of Environmental Performance Information - A Dual Test of Media Agenda Setting Theory and Legitimacy Theory", Accounting and Business Review, 29(1), 21-41.

CAMPBELL, J.L. (2006): "Institutional analysis and the paradox of corporate social responsibility", American Behavioral Scientist, 49(7), 925-938.

COMISIÓN NACIONAL DEL MERCADO DE VALORES (1998): "Informe de la comisión especial para el estudio de un código ético de los consejos de administración de las sociedades". Disponible en: http://www.cnmv.es/portal/legislacion/COBG/COBG.aspx.

COMISION NACIONAL DEL MERCADO DE VALORES (2003): "Informe de la comisión especial para el fomento de la transparencia y seguridad en los mercados y en las sociedades cotizadas". Disponible en: http://www.cnmv.es/portal/legislacion/COBG/COBG.aspx.

COMISION NACIONAL DEL MERCADO DE VALORES (2006): "Código Unificado de Buen Gobierno de las Sociedades Cotizadas o Código Conthe". Disponible en: http://www.cnmv.es/portal/legislacion/COBG/COBG.aspx.

COOPER, S. \& OWEN, D. (2007): "Corporate social reporting and stakeholder accountability: The missing link", Accounting, Organizations and Society, 32(7), 649-667.

CORPORATE REGISTRER (2013): "CRPerspectives 2013, Global CR Reporting Trends and Stakeholder Views". Disponible en: http://www.csrinternational.org/govresearch/global-crreporting-trends.

Corporate Knights Capital (2014): "Measuring Sustainability Disclosure: Ranking the World's Stock Exchanges". Disponible en: http://www.corporateknights.com/wpcontent/reports/2014_World_Stock_Exchange.pdf.

COWEN, S., FERRERI, L. \& PARKER, D. (1987): "The Impact of Corporate Characteristics on Social Responsibility Disclosure: A Typology and Frequency-Based Analysis", Accounting Organizations and Society, 12 (2), 111-22.

DANDO, N. \& SWIFT, T. (2003): "Transparency and assurance: Minding the credibility gap", Journal of Business Ethics, 44 (2/3), 195-200.

DEEGAN, C. \& GORDON, B. (1996): "A study of the environmental disclosure policies of Australian corporations", Accounting and Business Research, 26(3), 187-99.

DEEGAN, C. \& RANKIN, M. (1996): "Do Australian Companies Report Environmental News Objectively? An Analysis of Environmental Disclosures by Firms Prosecuted Successfully by the Environmental Protection Authority", Accounting, Auditing \& Accountability Journal, 9(2), 50-67. 
DEEGAN, C. \& RANKIN, M. (1997): "The materiality of environmental information to users of annual reports", Accounting, Auditing \& Accountability Journal, 10(4), 562-580.

DIMAGGIO, P. y POWELL, W. (1983): "The Iron Cage revisited: institutional isomorphism and collective rationality in organizational fields", American Sociological Review, 48(2), 147-160.

DIMAGGIO, P.J. \& POWELL, W.W. (Eds.). (1991): The new institutionalism in organizational analysis, Chicago, IL: University of Chicago Press.

EDELMAN (2008): "Edelman Trust Barometer 2008". Disponible en: http://www.edelman.com/assets/uploads/2014/01/2008-Trust-Barometer-Global-Results.pdf.

ELKINGTON, J. (1997): Cannibals with Forks: the Triple Bottom Line of 21st Century Business, Capstone Publishing Ltd, Oxford.

FIFKA, M (2012): "The development and state of research on social and environmental reporting in global comparison", Journal für Betriebswirtschaft, 62(1), 45-84.

FINANCIAL ACCOUNTING STANDARDS BOARD (1980): Statement of Financial Accounting Concepts No. 2: Qualitative Characteristics of Accounting Information, Financial Accounting Standards Board. Disponible en: http://www.fasb.org/cs/BlobServer?blobcol=urldata\&blobtable=MungoBlobs\&blobkey=id\&blobwhere=1175820900526\&blobheader=application\%2Fpdf.

GLOBAL REPORTING INICIATIVE (2004): "Guía para la elaboración de memorias de sostenibilidad, versión 3.0". Disponible en: http://www.observatorio-rse.org.es/Publicaciones/Guia.pdf.

GLOBAL REPORTING INICIATIVE (2011): "Guía para la elaboración de memorias de sostenibilidad, versión 3.1". Disponible en: https://www.globalreporting.org/resourcelibrary/Spanish-G3.1Complete.pdf.

GLOBAL REPORTING INICIATIVE (2015): "Sustainability and Reporting Trends in 2025". Disponble en: https://www.globalreporting.org/resourcelibrary/Sustainability-and-Reporting-Trends-in-20251.pdf.

GÓMEZ, N. \& LABURU, E. (2008): "Divulgación de información sobre empleados y medio ambiente en España y Alemania: una nota de investigación", Revista de contabilidad, 11(2), 123.

GOVERNANCE \& ACCOUNTABILITY INSTITUTE, INC (2015): Nota de prensa extraída de http://www.ga-institute.com/nc/issue-master-system/news-details/article/flash-report-seventy-fivepercent-75-of-the-sp-index-published-corporate-sustainability-rep.html.

GRAY, R. (2000): "Thirty years of social accounting, auditing and reporting: what (if anything) have we learned?", Business Ethics: a European Review, 10 (1), 9-15.

GRAY, R., DEY, C., OWEN, D., EVANS, R. \& ZADEK, S. (1997): "Struggling with the praxis of social accounting Stakeholders, accountability, audits and procedures", Accounting, Auditing \& Accountability Journal, 10 (3), 325-364. 
GRAY, R., KOUHY, R. \& LAVERS, S. (1995): "Corporate social and environmental reporting: A review of the literature and a longitudinal study of UK disclosure", Accounting, Auditing \& Accountability Journal, 8(2), 47-77.

GRAY, R., OWEN, D. \& ADAMS, C. (1996): Accounting and accountability: Changes and challenges in corporate social and environmental reporting, Prentice-Hall, London.

GRAY, R., OWEN, D. \& MAUNDERS, K. (1987): Corporate social reporting: accounting and accountability, PrenticeHall, London.

HACKSTON, D. \& MILNE, M.J. (1996): "Some determinants of social and environmental disclosures in New Zealand companies", Accounting, Auditing \& Accountability Journal, 9(1), 77-108.

HOFFER, G.E., PRUITT, S.W. \& REILLY, R.J. (1988): "The Impact of Product Recalls on the Wealth of Sellers: A Reexamination", Journal of Political Economy, University of Chicago Press, 96(3), 663-670.

HOGNER, R. (1982): "Corporate social reporting: Eight decades of development at US Steel", Research in Corporate Social Performance and Policy, 4, 243-250.

IDOWU, S. \& PAPASOLOMOU, I. (2007): "Are the corporate social responsibility matters based on good intentions or false pretences? An empirical study of the motivations behind the issuing of CSR reports by UK companies", Corporate Governance: The international journal of business in society, 7 (2), 136-147.

INTERNATIONAL INTEGRATED REPORTING COMMITTEE: http://integratedreporting.org/ Website visitada en Agosto 2015.

JAIRO, J., STELLA, M. \& ESCOBAR, N. (2013): "Aproximación a los factores que influyen en la divulgación de información sobre RSC en empresas de América Latina", Cuadernos de contabilidad, 14, Universidad Javeriana, 91-124.

JENNINGS, P. \& ZANDBERGEN, P. (1995): "Ecologically sustainable organizations: An institutional approach", Academy of Management Review, 20(4), 1015-1052.

KELLY, G. (1981): "Australian social responsibility disclosure: some insights into contempory measurement", Accounting and Finance, 21 (2), 97-104.

KPMG (2014): "The KPMG Survey of Corporate Responsibility Reporting 2013". Disponible en https://www.kpmg.com/Global/en/lssuesAndlnsights/ArticlesPublications/corporateresponsibility/Documents/corporate-responsibility-reporting-survey-2013-exec-summary.pdf

KPMG (2002-2013): KPMG, international survey of Corporate Social Responsibility.

KPMG \& GLOBAL REPORTING INICIATIVE (2013): "Carrots and Sticks: Sustainability Reporting Policies Worldwide-Today's Best Practice, Tomorrow's Trends". Disponible en https://www.globalreporting.org/resourcelibrary/Carrots-and-Sticks.pdf 
LARRINAGA, C., MONEVA, J.M., LLENA, F., CARRASCO, F. \& CORREA, C. (2002): Regulación contable de la información medioambiental. Normativa española e internacional, AECA, Madrid.

LIU, X. \& ANBUMOZHI, V. (2009): "Determinant factors of corporate environmental information disclosure: an empirical study of Chinese listed companies", Journal of Cleaner Production, 17(6), 593-600.

LYDENBERG, S., ROGERS, J. \& WOOD, D. (2010): From transparency to performance: Industrybased sustainability reporting on key issues, Hauser Center for Nonprofit Organizations.

MCWILLIAMS, A. \& Siegel, D. (2001): "Corporate Social Responsibility: A Theory of the Firm Perspective", The Academy of Management Review, (1), 117-127.

MILNE, M. \& GRAY, R. (2013): "W(h)ither ecology? the triple bottom line, the global reporting initiative, and corporate sustainability reporting", Journal of Business Ethics, 118(1), 13-29.

MILLER, P., KURUNMAKI, L. \& O'LEARY, T. (2008): "Accounting, hybrids and the management of risk", Account Organ Soc, 33 (7/8), 942-967.

MONEVA, J.M. \& LLENA, F. (1996): "Análisis de la información sobre responsabilidad social en las empresas industriales que cotizan en bolsa", Revista Española de Financiación y Contabilidad, 361-402.

MONEVA, J.M. \& LLENA, F. (2000): "Environmental Disclosures in the Annual Reports of Large Companies in Spain", European Accounting Review, 9(1), 7-29.

NORMAN, W. \& MACDONALD, C. (2004): "Getting to the bottom of Triple Bottom Line", Business Ethics Quarterly, 14, 243-262.

NORTH, D. (1998): "Desempeño económico a través de los años", Economía: Teoría y Práctica, 9 , 127-138.

OBSERVATORIO DE PUBLICIDAD (2011): "Informe de Endesa". Disponible en: http://www.fundacion-ipade.org/observatorio-publicidad/wp-content/uploads/2011/06/Informe-ENDESA.pdf.

OBSERVATORIO DE PUBLICIDAD (2011): "Informe de Iberdrola". Disponible en: http://www.fundacion-ipade.org/observatorio-publicidad/wp-content/uploads/2011/08/Informe-lberdrola.pdf.

OBSERVATORIO DE PUBLICIDAD (2011): "Informe de Unión Fenosa". Disponible en: http://www.fundacion-ipade.org/observatorio-publicidad/wp-content/uploads/2011/08/Informe-UNION-FENOSA.pdf.

OBSERVATORIO DE RESPONSABILIDAD SOCIAL CORPORATIVA (2004-2012): "La responsabilidad social corporativa en las memorias anuales de las empresas del IBEX 35, análisis del ejercicio", Ejercicios 2003- 2011. Disponible en: www.observatoriorsc.org.

OCDE (1997): "Convenio de Lucha contra la Corrupción de Agentes Públicos Extranjeros en las Transacciones Comerciales Internacionales o Convenio Anticohecho". Disponible en: http://www.oecd.org/daf/anti-bribery/ConvCombatBribery_Spanish.pdf. 
O'DONOVAN, G. (2002): "Environmental disclosures in the annual report: Extending the applicability and predictive power of legitimacy theory", Accounting, Auditing \& Accountability Journal, 15(3), 344-371.

O'DWYER, B. \& OWEN, D.L. (2005): "Assurance statement practice in environmental, social and sustainability reporting: a critical evaluation", The British Accounting Review, 37(2), 205-229.

ORIJ, R. (2010): "Corporate social disclosures in the context of national cultures and stakeholder theory", Accounting, Auditing \& Accountability Journal, 23(7), 868-889.

OWEN, D., SWIFT, T. \& HUNT, K. (2001): "Questioning the role of stakeholder engagement in social and ethical accounting, auditing and reporting", Accounting Forum, 25(3), 264-282.

PANG, Y.H. (1982): "Disclosures of corporate social responsibility", The Chartered Accountant in Australia, 32-4.

PATTEN, D. (1991): "Exposure, legitimacy, and social disclosure", Journal of Accounting and Public Policy, 10, 297-308.

PATTEN, D. (1992): "Intra-industry environmental disclosures in response to the Alaskan oil spill: a note on legitimacy theory", Accounting, Organizations and Society, 17(5), 471-5.

PRAKASH (2015): "Report on the Scope and Quality of CSR Reports from the World Largest Companies", CSR-Sustainability Monitor y Weissman Center for International Business Baruch College, The City University of New York.

PONCE, H.G. \& LABAT, B. N. (2011): "La revelación de información social por parte de las empresas españolas: Factores explicativos y necesidad de legitimidad social", Contabilidad y Negocios, 6(12), 5-25.

RAMBAUD, A. \& RICHARD, J. (2015): "The 'Triple Depreciation Line' instead of the 'Triple Bottom Line": Towards a genuine integrated reporting', Critical Perspectives on Accounting, 1045-2354.

RAMIRO, P., GONZALEZ, E. \& PULIDO, A. (2007): La energía que apaga Colombia: los impactos de las inversiones de Repsol y Unión Fenosa, Icaria Editorial.

REPSOL (2011): "Informe Anual de Responsabilidad Social Corporativa, 2011". Disponible en http://www.repsol.com/imagenes/es_es/Informe_RC_2011_tcm7-625740.pdf.

REVERTE, C. (2009): "Determinants of corporate social responsibility disclosure ratings by Spanish listed firms", Journal of Business Ethics, 88(2), 351-366.

REVERTE, C. (2012): "The impact of better corporate social responsibility disclosure on the cost of equity capital", Corporate Social Responsibility and Environmental Management, 19(5), 253-272.

ROBERTS, R. (1992): "Determinants of Corporate Social Responsibility Disclosure: An Application of Stakeholder Theory", Accounting, Organizations and Society, 17(6), 595-612. 
ROGERS, J. (2013): "4 signs of sustainability from oil, gas and mining companies". [Web log comentario]. Extraído el 14 de Agosto de 2015 de http://www.greenbiz.com/blog/2013/11/05/4-sustainability-trends-oil-gas-mining.

SAM y PWC (2011): "The Sustainability Yearbook 2011". Disponible en http://www.compromisorse.com/upload/estudios/000/118/samSustainabilityYearbook2011.pdf

SOX (2002): "Sarbanes-Oxley Act of 2002”, Pub. L. No. 107-204, 116 Stat. 745 (30 de julio de 2002).

SCHULTZ, F., CASTELLO, I. \& MORSING, M. (2013): "The construction of corporate social responsibility in network societies: A communication view", Journal of Business Ethics, 115(4), 681-692.

SODERSTROM, N (2013): "Sustainability reporting: past, present, and trends for the future", Insights Melburrne buseness and economics, 13, 31-37.

SPENCER, B. \& TAYLOR, G. (1987): "A Within and Between Analysis of the Relationship between Corporate Social Responsibility and Financial Performance", Akron Business and Economic Review, 18(3), 7-18.

SUCHMAN, M. (1995): "Managing Legitimacy: Strategic and Institutional Approaches", Academy of Management Review, 20(3), 571-610.

SUSTAINABILITY \& UNEP (1997): "Sustainability 1997 benchmark survey". Disponible en: http://www.sustainability.com/library/the-1997-benchmark-survey.

SUSTAINABILITY ACCOUNTING STANDARDS BOARD (SASB): http://www.sasb.org/, Webasite visitada el 16 de agosto de 2015.

TAGESSON, T., BLANK, V., BROBERG, P. \& COLLIN, S.O. (2009): "What explains the extent and content of social and environmental disclosures on corporate websites: a study of social and environmental reporting in Swedish listed corporations", Corp Social Responsibility and Environmental Management, 16, 352-364.

THE BUSINESS FOR A BETTER WORLD Y GLOBESCAN (2011): "State of Sustainable". Disponible en: www.bsr.org/.

THE BUSINESS FOR A BETTER WORLD Y GLOBESCAN (2014): "State of Sustainable". Disponible en: www.bsr.org/.

TEOH, H.Y. \& THONG, G. (1984): "Another look at corporate social responsibility and reporting: an empirical study in a developing country", Accounting, Organizations and Society, 9(2), 189-206.

THOMSON, I. (2015): "But does sustainability need capitalism or an integrated report? a commentary on 'The International Integrated Reporting Council: A story of failure"', Critical Perspectives on Accounting, 27, 18-22. 
ULLMAN, A. (1985): "Data in search of a theory: A critical examination of the relationships among social performance, social disclosure, and economic performance of US firms", Academy of management review, 10(3), 540-557.

WEISSMAN CENTER FOR INTERNATIONAL BUSINESS AT BARUCH COLLEGE (2014): "Report on the Scope and Quality of CSR Reports from the World's Largest Companies". Disponble en: http://www.csrsmonitor.org/archive/CSR-S\%20Monitor\%202014\%20Report.pdf.

WHEELER, D. \& SILLANPAA, M. (1998): "Including the stakeholder: The business case", Long Range Planning, 31(2), 201-210. 
